\title{
Using a Cold Radiometer to Measure Heat Loads and Survey Heat Leaks
}

\author{
M. DiPirro, J. Tuttle, T. Hait, and P. Shirron \\ Cryogenics and Fluids Branch, NASA/Goddard Space Flight Center, Greenbelt MD 20771 USA
}

\begin{abstract}
We have developed an inexpensive cold radiometer for use in thermal/vacuum chambers to measure heat loads, characterize emissivity and specularity of surfaces and to survey areas to evaluate stray heat loads. We report here the results of two such tests for the James Webb Space Telescope to measure heat loads and effective emissivities of 2 major pieces of optical ground support equipment that will be used in upcoming thermal vacuum testing of the Telescope.
\end{abstract}

\section{INTRODUCTION}

\section{Cold Radiometers}

Low cost radiometers were developed to allow in situ measurements of heat flux from both known and unknown sources in thermal-vacuum tests. These radiometers utilize a Winston cone to concentrate incident radiation from a relative compact solid angle onto an absorber and attached thermometer. The absorber and thermometer are weakly coupled to the base of the radiometer on which another thermometer is attached to compare temperatures. The difference between the heated sensor thermometer and the base thermometer, when multiplied by the temperature and gain factor, gives the heat load captured by the radiometer. This, in turn, by knowledge of the emissivity of the absorber and acceptance angle of the Winston cone, gives the equivalent flux emanating from the source. The lower the operating temperature, the more sensitive the thermometers and the better the thermal isolation. The type of thermometers used, CernoxTM 1080, give the best resolution in the 15$25 \mathrm{~K}$ range. More details of the design, testing and calibration are given in References 1 and 2. Key features of the radiometers are that the response to signals entering within the angle of acceptance $\left(11^{\circ}\right.$ half angle field of view (FOV)) is flat and the absorbing material, "Steelcast" is wavelength independent [3] over the blackbody radiation spectrum of interest in these tests. These properties greatly simplify the calibration and interpretation of the radiometer signals.

The radiometers can be calibrated in situ by applying extra electrical power to the thermometer and reading out the apparent temperature. The same is done for the base thermometer to compensate for the self-heating within the sensor itself. A plot of input power vs. temperature difference between the sensor and the base then produces the gain of the radiometer.

\section{JWST Testing}

The James Webb Space Telescope (JWST) is a $6.7 \mathrm{~m}$ diameter visible and infrared telescope that will be operated in the 40-60 K range in space. To verify proper operation, the telescope and instruments will be tested in large thermal-vacuum chambers. (See, for instance, FIGURE 1.) These tests are very expensive, so to reduce risk, several pieces of critical Ground Support Equipment (GSE) to be used for these tests are characterized for optical and thermal performance. The radiometer-related aspects of two of these GSE thermal tests will be described in this paper. A third major piece of optical GSE, the Cryogenic Photogrammetry Module was also tested using cold radiometers. Results of that test will be reported later. 


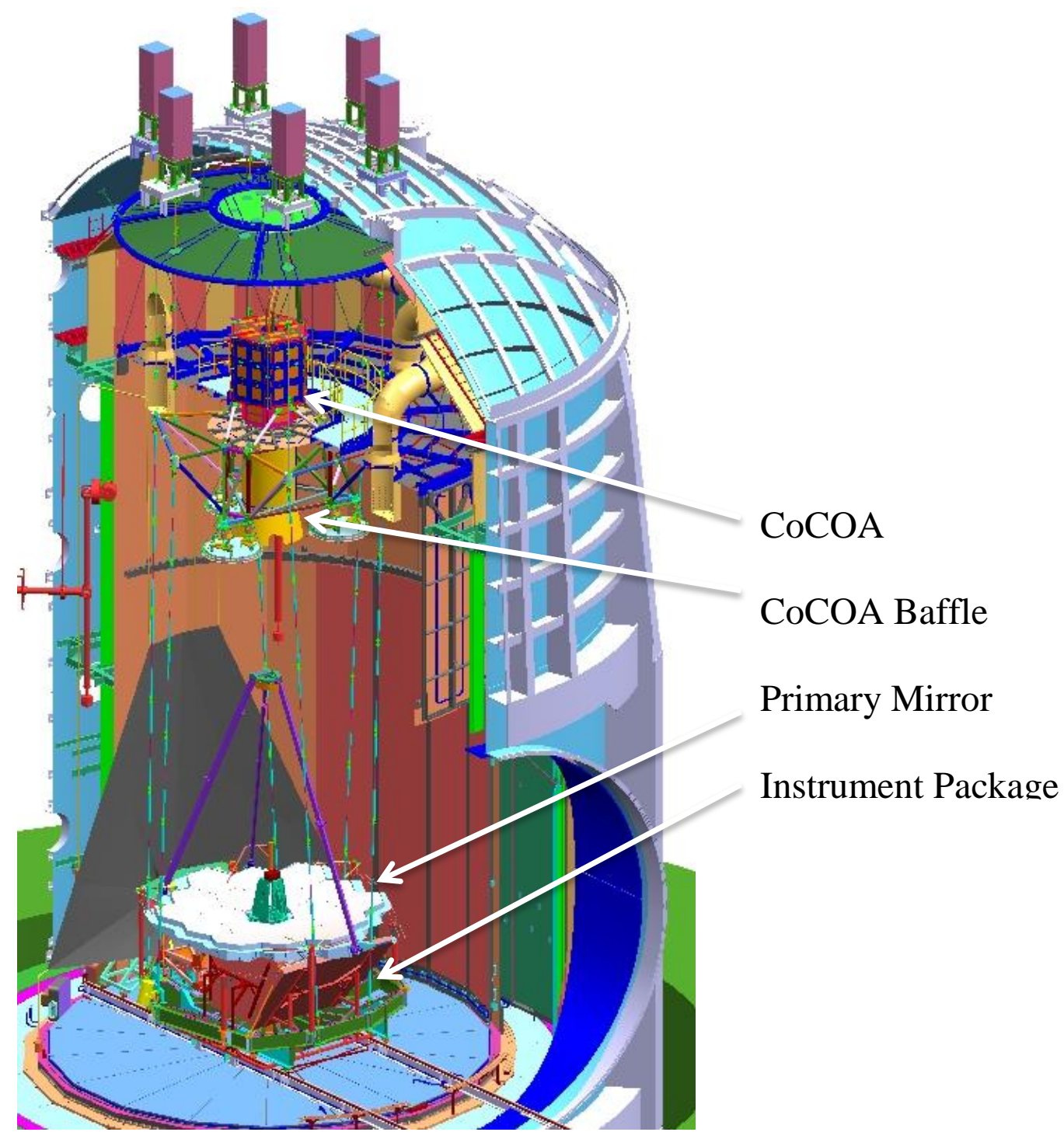

FIGURE 1. Test configuration of the JWST telescope and instrument package at Johnson Space Flight Center, Chamber A. For scale, the Primary mirror is 6.7 meters in diameter.

\section{COCOA TESTS}

One of the pieces of critical GSE is the Center of Curvature Optical Assembly (CoCOA). This large interferometer operates outside the thermal vacuum chamber at room temperature but has a view into the chamber through a large glass port. To protect thermal balance testing, a passively cooled shutter is placed in front of the window when the CoCOA is not being used. It was necessary to measure the shutter temperature and thermal emissivity and the emission from the CoCOA itself in two configurations with the shutter open

A test of the CoCOA was performed at Marshall Space Flight Center's large $20 \mathrm{~K}$-shrouded thermal vacuum chamber know as the XRCF. While the CoCOA itself was within a surrounding $120 \mathrm{~K}$ shroud, it operates at room temperature and in normal room temperature air. It was therefore surrounded by a hermetic shell and by MLI and kept warm by the flow of air ducted from outside the chamber. A plate cooled by a dedicated $20 \mathrm{~K}$ refrigerator cryogen loop was mounted directly under the CoCOA. Four radiometers were placed on this plate as shown in FIGURE 2 under the CoCOA shutter. The radiometers had an additional cylinder surrounding the Winston cone to prevent stray radiation from a warm $(120 \mathrm{~K})$ part of the shroud from heating the body of the 
radiometer. An additional radiometer was positioned on the cold plate to look directly at this hot part of the shroud.

The shutter was constructed with an aluminum frame with an MLI blanket. It was thermally isolated from the room temperature CoCOA and was allowed to float thermally between the warm CoCOA above and the cold chamber below. On the bottom of the shutter, the last, outward facing layer was black kapton with VDA aluminum on the blanket side. It was this layer that the radiometers faced. Radiometer \#4, R4, faced the the center of this outer blanket. A Cernox thermometer was taped to the outside of the blanket and covered with a small patch of black kapton to match the emissivity of the rest of the shutter. By measuring the temperature the radiometer reading could be converted directly to an effective emissivity.

The shutter was allowed to cool to its equilibrium temperature over several days. The cold plate below the shutter was maintained at less than $24 \mathrm{~K}$ during this time. Radiometer measurements were made continuously. All 4 radiometers viewing the shutter game the same reading to better than $\pm 5 \%$. The shutter temperature reached $103.4 \mathrm{~K}$ and from the $\mathrm{R} 4$ reading its emissivity was 0.62 , which is slightly lower than the expected value of 0.74 at this temperature. Perhaps the aluminized surface provided a reflection of any transmitted radiation. The total emission per area from the shutter bottom surface was $4000 \mathrm{~mW} / \mathrm{m}^{2}$, which, in turn is used to calculate the amount of power escaping into the chamber to be $0.27 \mathrm{~W}$, which is within the acceptable range for its final use in the JWST test.

The shutter was then opened. Two modes of the CoCOA with different thermal signatures were tested: one with a hologram in place which has blanketing limiting the total heat output, and one with the hologram retracted which had the signature of a $293 \mathrm{~K}$ blackbody. For this mode the heat load out of the aperture was $27.8 \mathrm{~W}$.

These tests were repeated several times for different durations over several weeks to simulate expected operations in Chamber A and to test the thermal limits and transient response.

The radiometers were not initially designed to stare at a heat source as large as room temperature over the entire FOV. To obtain accurate results when the radiometer was operated with a very high heat load, it was necessary to calibrate the same radiometer a second time in the presence of this high heat load. The same calibration procedure as performed at low temperature was followed, but higher electrical power was used. The temperature-dependence curve that was used for readout between 15 and $25 \mathrm{~K}$ was surprisingly relatively accurate even when the sensing element was at $75 \mathrm{~K}$, and followed the thermal conductance dependence of stainless steel as expected. This gave confidence in the accuracy of the heat load measurements.

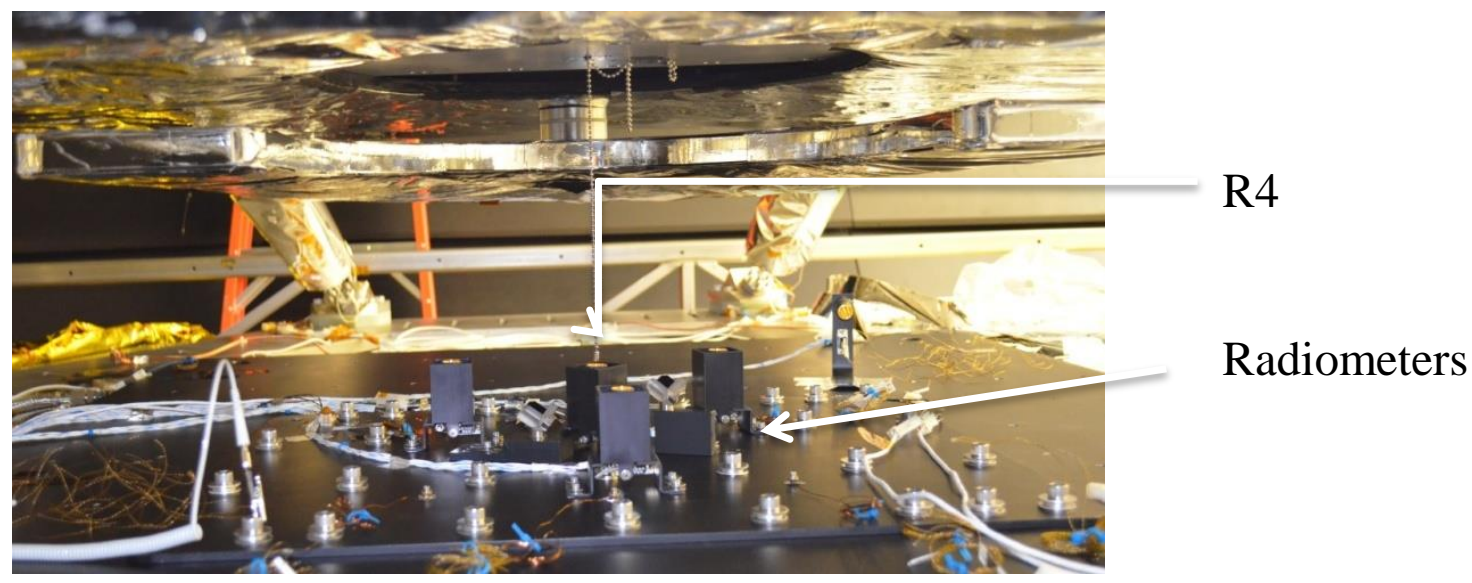

FIGURE 2. Four radiometers (R4 is back center) mounted on the cold plate under the CoCOA shutter. The shutter has a "U" shaped opening for balancing and is in the open position in this picture. 


\section{OSIM TESTS}

\section{Objectives}

Before integrating the Integrated Science Instrument Module (ISIM) to the telescope, ISIM is tested by itself in a smaller thermal-vacuum chamber at Goddard Space Flight Center (GSFC). A smaller, simulator telescope, the Optical Simulator for the Instrument Module (OSIM) is used to test the instruments. OSIM was cryogenically tested before installation of ISIM, characterizing its optical and thermal performance. The thermal performance tests included a measurement of the heat load into ISIM (nominal $40 \mathrm{~K}$ ) from OSIM (nominal 100 K). Compared to the JWST primary, secondary and tertiary mirrors, OSIM emits a significant amount of heat -$100 \mathrm{~K}$ vs. $30-50 \mathrm{~K}$ for the flight mirrors. An important measurement to be made in the ISIM test is the lead load balance; measure heat into ISIM through harnesses, structure and internal dissipation to determine if the passive radiators have sufficient cooling power to maintain the instruments at their operating temperature (typically 36$40 \mathrm{~K}$ ). To limit the amount of heat entering the ISIM cavity from OSIM a baffle is used. The baffle is a slightly tapered, rectangular cross section, duct whose inside is painted with Ball InfraRed Black (BIRB) which has a high emissivity and low specularity.

The goals of the radiometer measurements were to measure the heat load that will radiate from the OSIM into the ISIM cavity, determine the effectiveness of the baffle in limiting this heat load, and determine the effective emissivity of the OSIM aperture

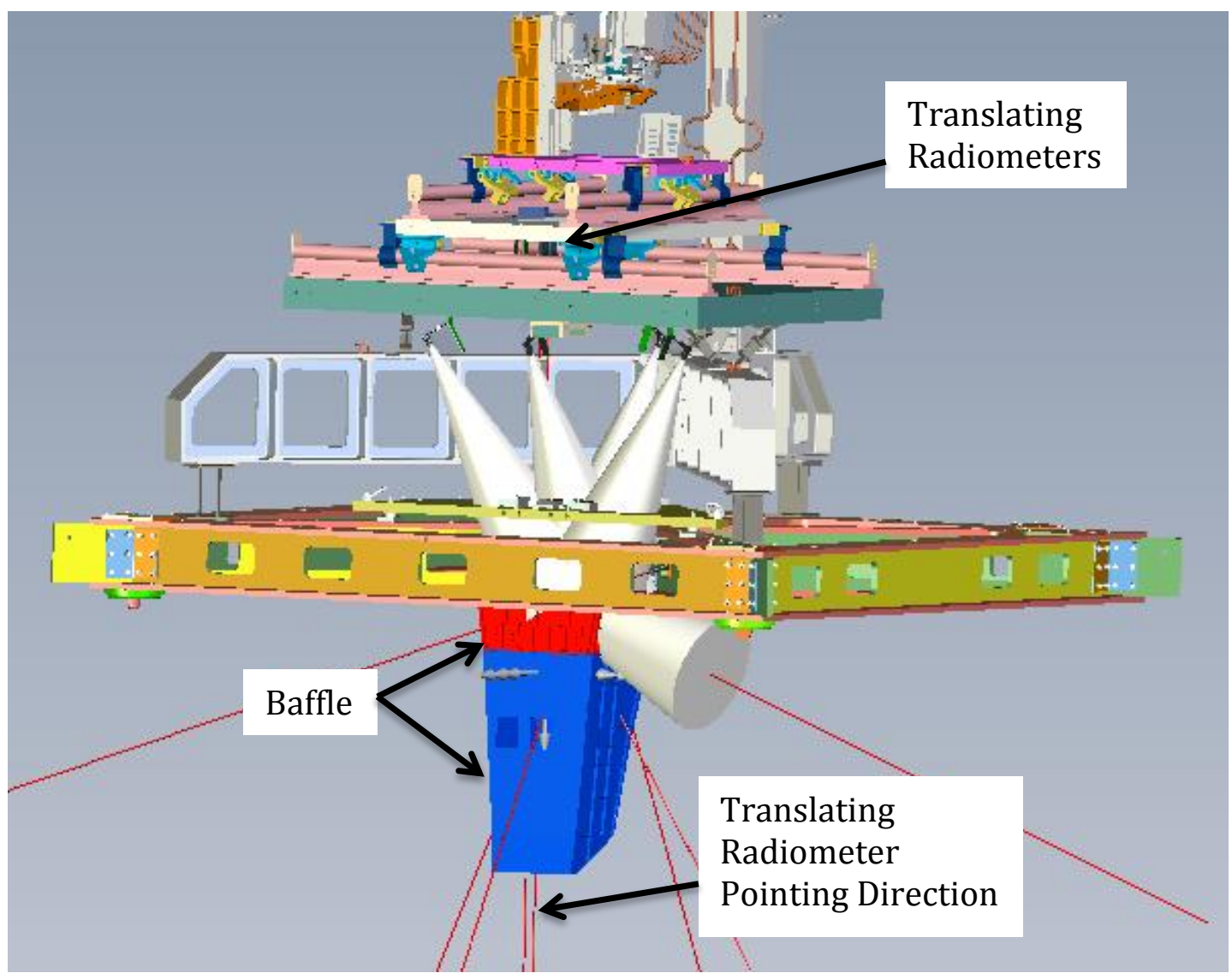

FIGURE 3. Three dimensional drawing of the OSIM test apparatus, showing the baffle and FOVs of the fixed radiometers (gray cones). The radiometers' central axes are shown as straight lines. The two parallel lines at the bottom of the baffle are from the two translating radiometers. 


\section{Set Up}

Six radiometers were stationary, positioned around the exit aperture two of which had a view of only the upper portion of the baffle and four of which had views of the four walls of the baffle. None of these radiometers had a view of the lowest $20 \%$ of the baffle or the aperture. Two radiometers were attached to a moveable stage that translated across the baffle, aperture, and a support structure. These two radiometers pointed directly down. (See Figures 3 and 4.) Unfortunately all radiometers but the lowest two were mounted to structures that were not well anchored to the $15 \mathrm{~K}$ shroud, and due to dissipation by the optical GSE attached to the moveable structure, operated at temperatures between 34-47 K for the first test, and 29-36 K in the second test. The higher than expected temperatures had two adverse effects: They limited the resolution (signal to noise) of the radiometers, and caused a longer that expected time constant of 5-10 minutes rather than the expected 10-20 seconds. Part of this longer time constant was due to the structure itself relaxing rather than the radiometer. The two radiometers viewing only the very upper portion of the baffle were properly anchored at 17 $\mathrm{K}$ and had excellent signal to noise. These demonstrated that no noticeable reflections $\left(<5 \mathrm{~mW} / \mathrm{m}^{2}\right)$ came from the upper baffle. The moveable radiometers were located $1.11 \mathrm{~m}$ above the exit of the baffle. For this reason, their FOV included multiple elements as is described in the next section. The baffle geometry itself was not symmetric due to the last folding flat mirror which accepted light from the side (FIGURE 4). Radiometer \#9, the more sensitive of the two movable radiometers is close to the center of the baffle, but is not also centered over the aperture.

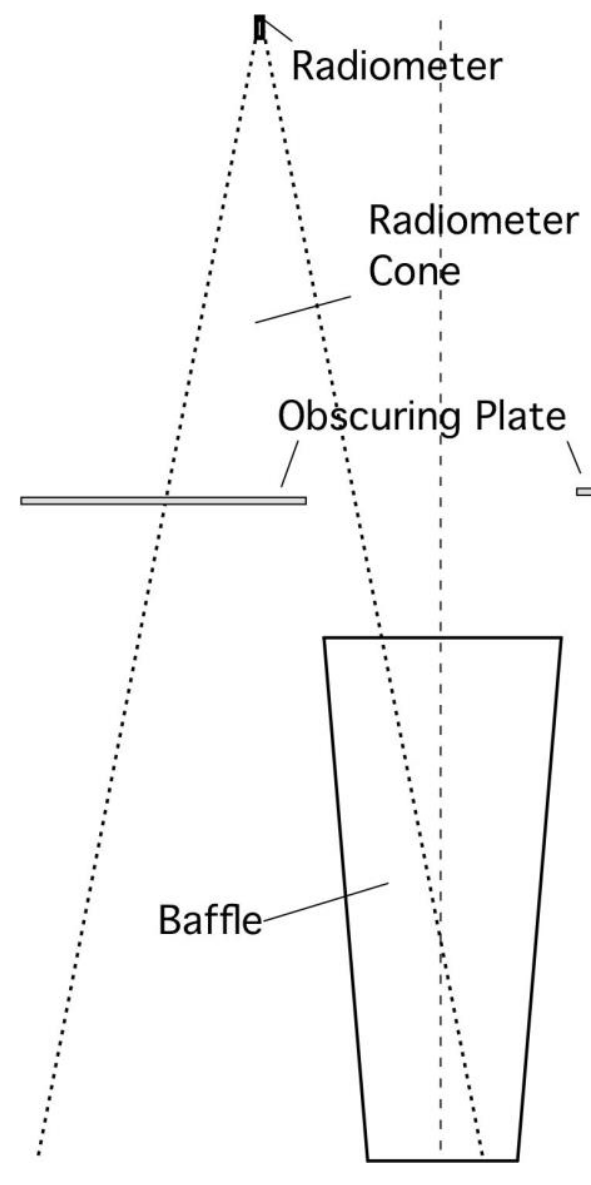

(a)

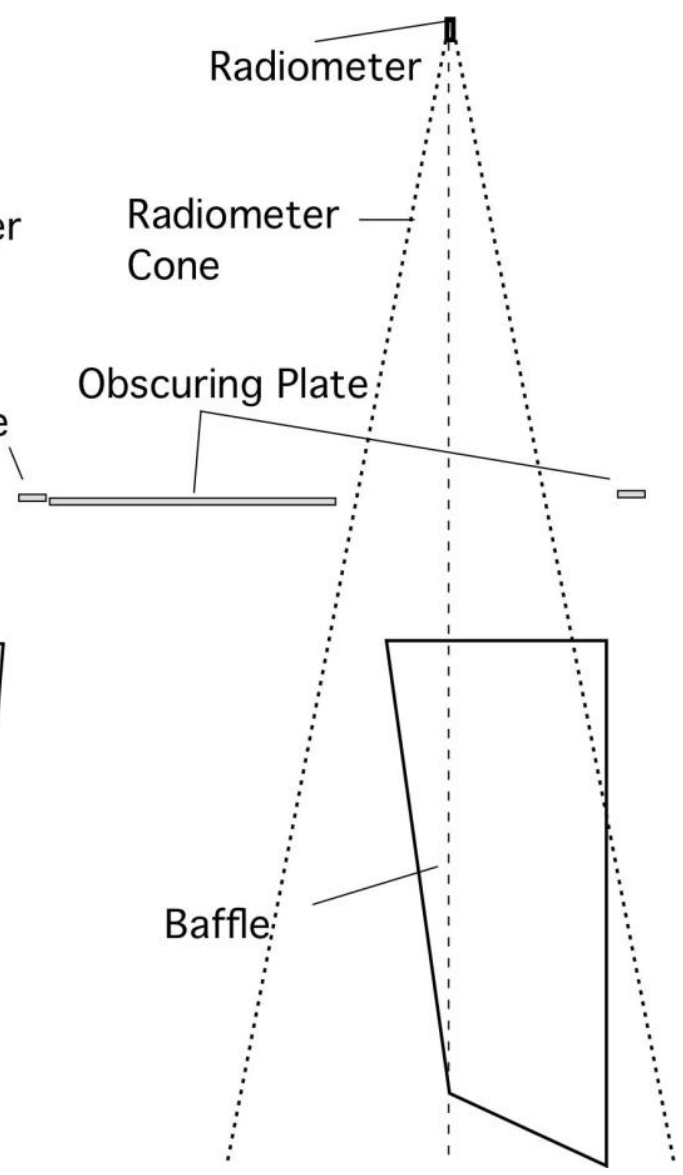

(b)

FIGURE 4. A schematic elevation of the baffle, obscuring plate, and radiometers. The radiometer cone is $11^{\circ}$ half angle. (a) The radiometer position shown is $320 \mathrm{~mm}$ to the left of the center position. (b) view in direction of travel indicating the lack of symmetry in the baffle entrance. 


\section{Results and Analysis}

The measurements performed on the OSIM suffered from the number of different thermal emitters in the field of view. Heat from the optical instrument also appeared as a reflection off some of the neighboring surfaces visible to the radiometer, which confused the results. The source of this heat was traced to warm (120 to $140 \mathrm{~K})$ preamplifier boxes located next to the optical and near infrared cameras located above the back of the radiometers. These boxes had a direct line of sight to surface $\mathrm{C}$ in FIGURE 5. The boxes were not able to be turned off and allowed to cool, but changing their temperature from 140 to $120 \mathrm{~K}$ reduced the signal background by nearly a factor of two. When the radiometer is centered over the exit of the baffle, the aperture is off to one side. The view is represented by the schematic in FIGURE 5. Interpretation of this scene is ambiguous with only one view. To help deconvolve the radiation coming from each of the sources, the radiometer was translated across the exit of the aperture in the direction indicated in FIGURE 6. Near the edge of the baffle the change in signal was greatest, so the radiometer was moved in $25 \mathrm{~mm}$ steps. The FOV was divided into 5 zones as indicated in FIGURE 5. For simplicity, a constant flux per unit area was assigned to each zone. We compare the proportion of the FOV of each of the items multiplied by a flux per unit area for each item to come up with a predicted value for the flux into the radiometer. This result is compared to the actual reading. A best fit to the data is obtained by varying each of the items' flux intensity. The result of this analysis is shown in FIGURE 7. Once the flux intensity is obtained, the heat load is determined by multiplying by each item's view factor. A more thorough analysis of the view factors will be done in the future to better fit the measured values.

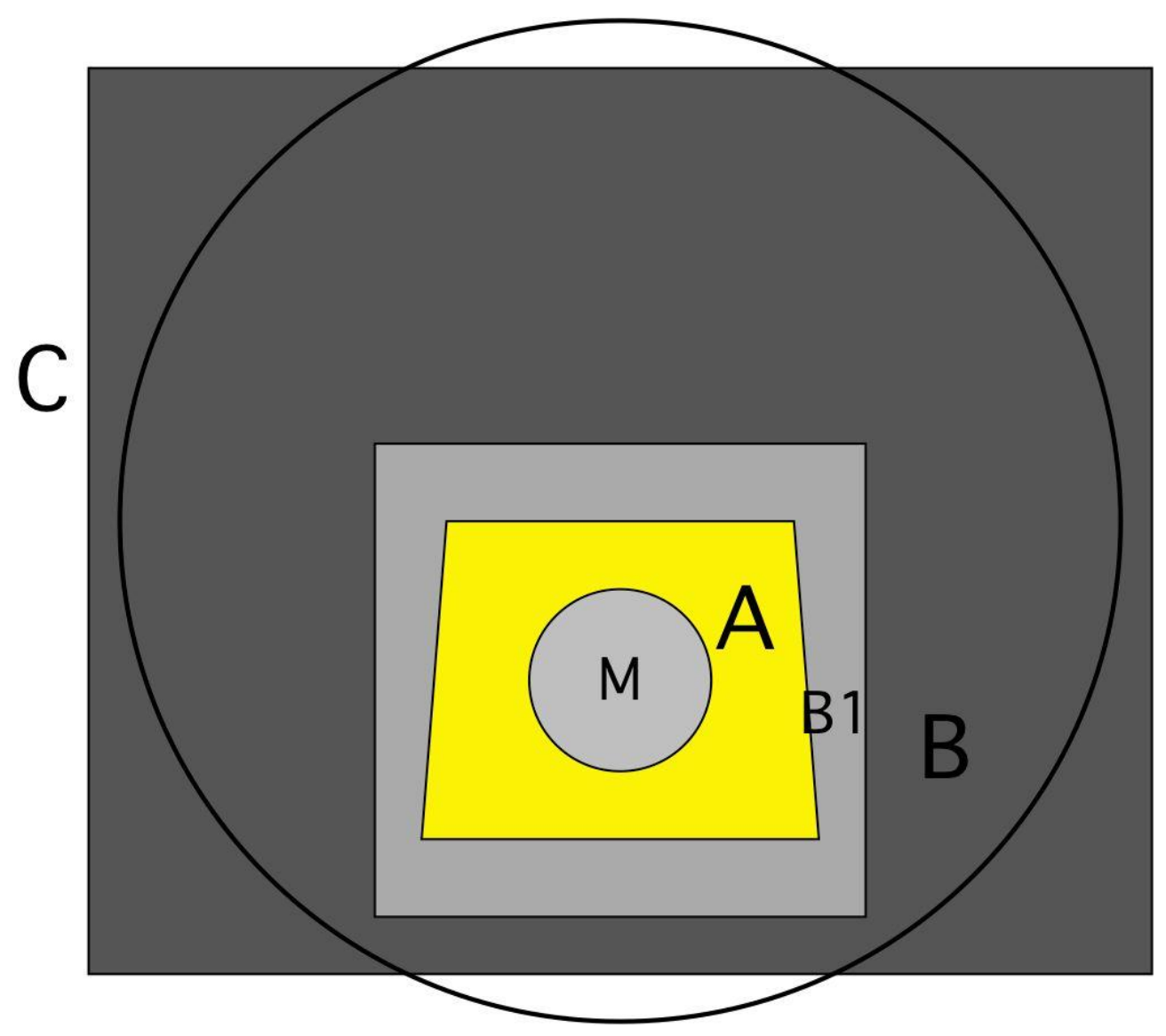

FIGURE 5. Depiction of the scene within the radiometer FOV. Area $A$ is the aperture to the OSIM mirror, $M$ is the mirror chain, B is the upper $80 \%$ of the baffle, B1 is the lower $20 \%$ of the baffle, and C is the obscuring plate and other areas above the exit of the baffle. The circle is the radiometer field of view. 


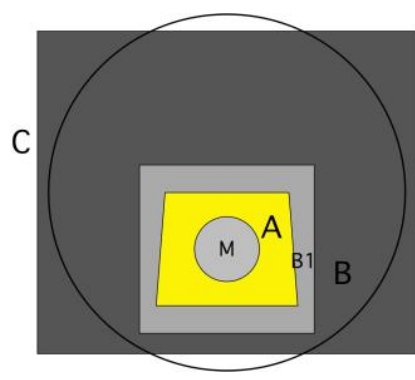

(a)

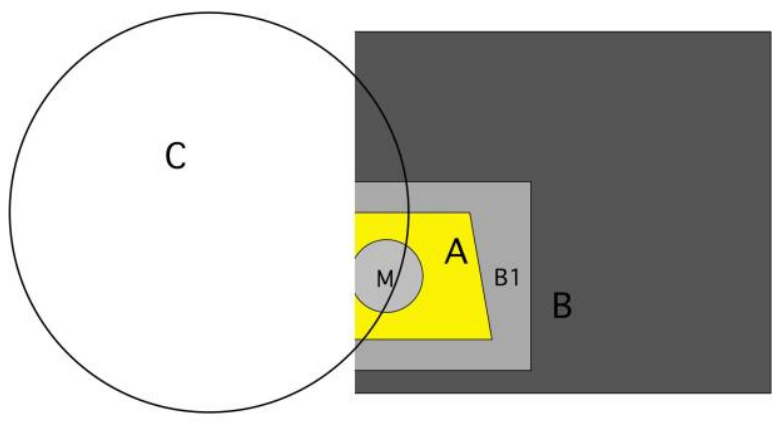

(d)

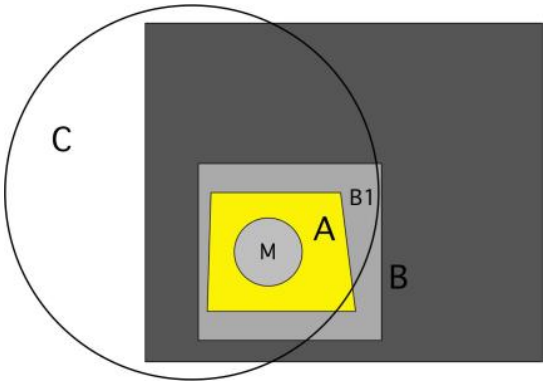

(b)

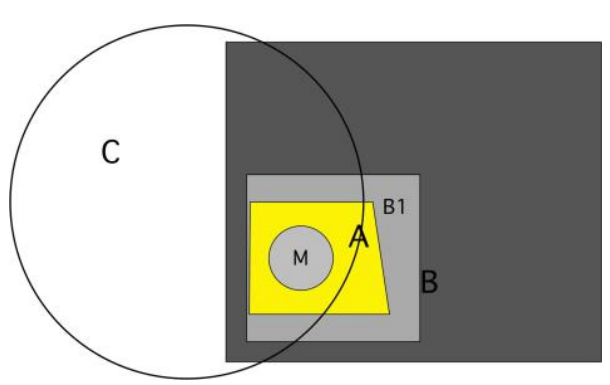

(c)

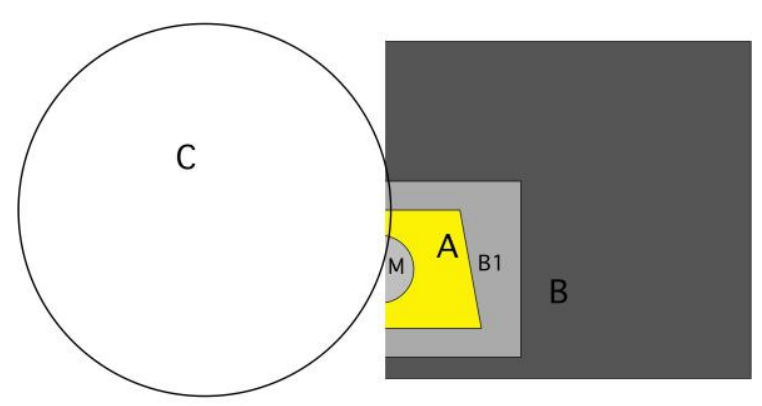

(e)

FIGURE 6. Progression of the FOV as the radiometer translates from the center to the left side of the baffle and aperture. (a) centered, (b) $173 \mathrm{~mm}$ left, (c) $273 \mathrm{~mm}$ left, (d) $348 \mathrm{~mm}$ left, (e) $398 \mathrm{~mm}$ left. Note that items moving to the right are in the foreground and items moving to the left are in the background relative to the center of the entrance aperture, A.

Early in the testing we obtained the effective emissivity of the aperture's mirror chain by making a measurement with and without a blocking "filter" at the pupil. This filter had a black kapton outer surface which had an expected emissivity of 0.74 . The difference in the measured heat at the radiometer combined with the known fractional solid angle of the pupil gave an effective emissivity of the mirror chain of 0.085 . This value is consistent with a chain of 4 mirrors each with an emissivity of $<0.03$, as expected. This knowledge, in turn, was used to fix the heat flux from area M. Also, since the radiometer readings remained constant when the radiometers were moved more than $400 \mathrm{~mm}$ from the center, this value $\left(196 \mathrm{~mW} / \mathrm{m}^{2}\right)$ was taken to be the value for $\mathrm{C}$.

For OSIM we obtained a total heat load of $21.5 \mathrm{~mW} \pm 2.0 \mathrm{~mW}$ through the exit of the baffle. This consists of $20.4 \mathrm{~mW}$ from the entrance aperture to the baffle, $0.5 \mathrm{~mW}$ from the mirror chain, and $0.6 \mathrm{~mW}$ from reflections from the baffle, primarily from the area near the entrance aperture. The total power is relatively insensitive to the portion coming from reflections vs. from the aperture directly. The result compares favorably to the conservatively estimated $28 \mathrm{~mW}$ in the baffle and aperture design. The primary contributors to the error bar are uncertainty in the location of baffle and obscuring plate reflections, and the absolute accuracy of the radiometer calibration. It became apparent when fitting the data that the calculated data are very sensitive to certain radiometer positions. This is good because it restricts the possible interpretation of the measured data. However this also means that the calculated values are very sensitive to the real positions of the elements in the scene. For instance, the fit is not good in the range from -150 to $-200 \mathrm{~mm}$. This is probably due to inaccuracy of the modeling of the edge of the obscuring plate. A $20 \mathrm{~mm}$ change in position of this obscuring plate would provide a good fit to the data. 


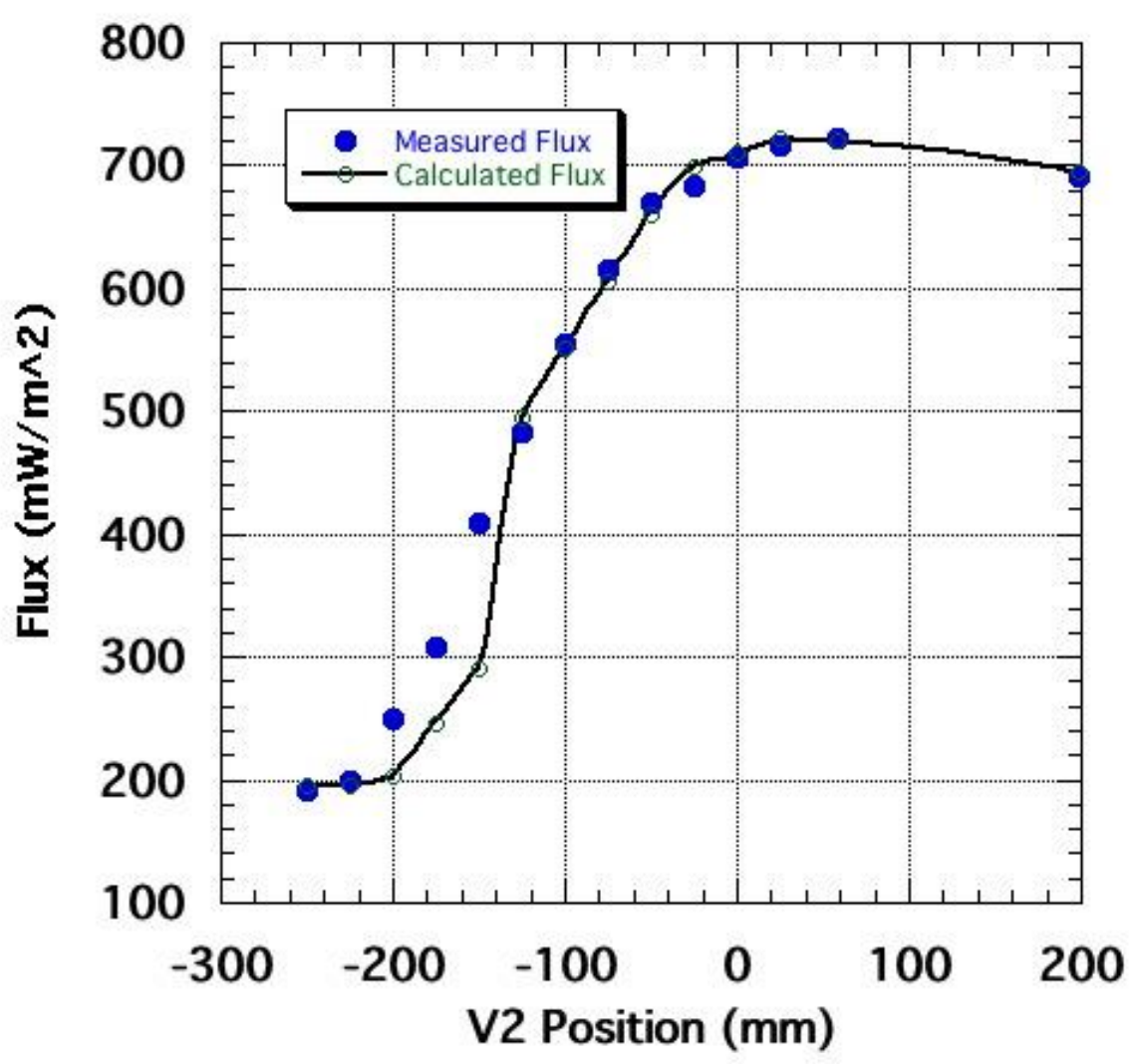

FIGURE 7. The radiometer measurements and calculated results for OSIM. Note that the line is a guide to the eye for the calculated values and is not a fit to the data. The error bars on the measurements are approximately the size of the filled circles.

\section{SUMMARY}

This paper describes two tests using the cold radiometers developed at NASA. The results demonstrate the usefulness of the radiometers in determining radiative heat fluxes, and effective emissivities. Even when a scene has several different emitters within it, by varying the radiometer position and/or the temperature of the sources, knowledge of the flux from each item can be obtained.

\section{REFERENCES}

1. M. DiPirro, T. Hait, J. Tuttle, and E. Canavan, "A low cost, low temperature radiometer for thermal measurements", Proc. SPIE 7439C-44 (2009).

2. DiPirro, M., Tuttle, J., Canavan, E., and Shirron, P., "Use of Cold Radiometers in Several Thermal/Vacuum Tests", Adv. Cryo. Eng. 57, (2012) pp. 1513-1518.

3. E. J. Wollack, D. J. Fixsen, R. Henry, A. Kogut, M. Limon, and P. Mirel, "Electromagnetic and Thermal Properties of a Conductively Loaded Epoxy”, Int. J. Infrared and Millimeter Waves (2008) 29:51-61. 